\title{
Gene expression in archived newborn blood spots distinguishes infants who will later develop cerebral palsy from matched controls
}

\author{
Nhan Thi Ho', Kyle Furge ${ }^{2}$, Wenjiang Fu', Julia Busik³, Sok Kean Khoo ${ }^{4}$, Qing Lu', Madeleine Lenski', Julia Wirth', \\ Edward Hurvitz ${ }^{5}$, Nancy Dodge ${ }^{6}$, James Resau ${ }^{4}$ and Nigel Paneth ${ }^{1,7}$
}

BACKGROUND: Gene expression in archived newborn blood spots remaining from newborn screening may reflect pathophysiological disturbances useful in understanding the etiology of cerebral palsy (CP).

METHODS: We quantified the expression of gene sets representing four physiological pathways hypothesized to contribute to $C P$ in archived unfrozen residual newborn blood spot specimens from 53 children with CP and 53 age-, gender-, and gestational age-matched controls. We selected four empirical and three canonical gene sets representing the inflammatory, hypoxic, coagulative, and thyroidal pathways and examined mRNA expression using an $8 \times 60,000$ oligonucleotide microarray. The $\log _{2}$ fold change of gene expression between matched cases and controls was analyzed using the generally applicable gene set enrichment method.

RESULTS: The empirical inflammatory and empirical hypoxic gene sets were significantly downregulated in term-born CP cases $(n=33)$ as compared with matched controls $(P=0.0007$ and 0.0009 , respectively), whereas both gene sets were significantly upregulated ( $P=0.0055$ and 0.0223 , respectively) in preterm-born CP cases $(n=20)$. The empirical thyroidal gene set was significantly upregulated in preterm-born CP cases $(P=0.0023)$. CONCLUSION: The newborn blood spot transcriptome can serve as a platform for investigating distinctive gene expression patterns in children who later develop CP.

C erebral palsy (CP) is a neurological syndrome of onset in early childhood characterized by impaired control of motor function (1), with a prevalence of $\sim 2-3$ per 1,000 live births in Western countries (2). The etiology of conditions, such as $\mathrm{CP}$, that originate with exposures occurring in pregnancy and the perinatal period is difficult to study because our capacity to interrogate such exposures once disease is manifest years later is limited. We here report on the assessment of biological processes occurring around birth, via examination of the transcriptome in residual filter paper blood spots archived after newborn genetic screening in 53 singleton children with CP and 53 age-, gender-, and gestational age-matched controls.

Examination of the transcriptome has found little application thus far in etiologic epidemiology. mRNA is hard to retrieve from serum samples because ribonucleases and micro-RNAs degrade mRNA quickly once transcriptional activity is complete. Although blood is ordinarily collected in glass or plastic tubes, permitting such enzymatic activity to continue, newborn blood is directly spotted from a heel stick incision onto filter paper, completely drying at room temperature within $3 \mathrm{~h}(3,4)$. RNA degradation is an enzymatic process tightly controlled by ribonucleases, water-soluble enzymes that require one water molecule for each reaction (5). Drying of the sample interferes with this reaction and also limits access of ribonucleases to RNA.

White blood cells share $>80 \%$ of the transcriptome with at least nine organs, including the brain (6); $82 \%$ of the 13,961 genes expressed in the brain, according to the Uni Gene database (http://www.ncbi.nlm.nih.gov/unigene), are expressed in blood (7). We hypothesized that gene sets reflecting four pathophysiological pathways to CP (inflammatory, hypoxic, coagulative, and thyroidal) would be dysregulated shortly after birth in children later diagnosed with $\mathrm{CP}$ and that this dysregulation would be detectable in newborn blood spots. We also performed exploratory gene set analyses using a database of clinically relevant gene sets (8). The hypothesized gene sets were obtained by searching the literature, aiming for one canonical gene set (i.e., based on expert opinion (9)) and one experimental gene set (i.e., based on experimental findings) for each pathway. For coagulation, we could only find a canonical gene set.

\section{RESULTS}

\section{Comparability of Cases and Controls}

Cases and controls were similar in gestational age, a matching criterion, but fetal growth was somewhat reduced in cases. More cases were admitted to newborn intensive care and had lower Apgar scores. Mean day of blood spot collection did not

\footnotetext{
'Department of Epidemiology and Biostatistics, College of Human Medicine, Michigan State University, East Lansing, Michigan; ${ }^{2}$ Laboratory of Computational Biology, Van Andel Research Institute, Grand Rapids, Michigan; ${ }^{3}$ Department of Physiology, Michigan State University, East Lansing, Michigan; ${ }^{4}$ Laboratory of Microarray Technology, Program in Biospecimen Science, Van Andel Research Institute, Grand Rapids, Michigan; ${ }^{5}$ Department of Physical Medicine and Rehabilitation, University of Michigan, Ann Arbor, Michigan; ${ }^{6}$ DeVos Children's Hospital, Grand Rapids, Michigan; ${ }^{7}$ Department of Pediatrics and Human Development, College of Human Medicine, Michigan State University, East Lansing, Michigan. Correspondence: Nigel Paneth (paneth@msu.edu)
} 
Table 1. Characteristics of cases and controls at birth of child

\begin{tabular}{|c|c|c|c|c|}
\hline \multirow[b]{2}{*}{ Characteristic } & \multicolumn{2}{|c|}{37 wk and above } & \multicolumn{2}{|c|}{ Under 37 wk } \\
\hline & Cases $(n=33)$ & Controls $(n=33)$ & Cases $(n=20)$ & Controls $(n=20)$ \\
\hline \multicolumn{5}{|l|}{ Biological/clinical } \\
\hline Mean birth weight & $3,372 \mathrm{~g}( \pm 495)$ & $3,562 \mathrm{~g}( \pm 432)$ & $1,884 \mathrm{~g}( \pm 889)$ & $2,106 \mathrm{~g}( \pm 902)$ \\
\hline Fetal growth ratio & 0.99 & 1.04 & 0.95 & 1.01 \\
\hline Mean 5-min Apgar score & $8.2( \pm 1.6)$ & $8.8( \pm 0.6)$ & $7.5( \pm 1.4)$ & $8.5( \pm 0.7)$ \\
\hline$\%$ Admitted to NICU & 36 & 9 & 95 & 78 \\
\hline Age blood spot obtained & $1.7 \mathrm{~d}( \pm 1.9)$ & $1.3 d( \pm 0.5)$ & $1.5 \mathrm{~d}( \pm 1.7)$ & $1.2 \mathrm{~d}( \pm 0.9)$ \\
\hline \multicolumn{5}{|l|}{ Sociodemographic } \\
\hline$\%$ Black & 12 & 3 & 10 & 20 \\
\hline$\%$ Home ownership & 86 & 68 & 59 & 71 \\
\hline$\%$ Medicaid support & 21 & 6 & 25 & 35 \\
\hline
\end{tabular}

Table 2. $\gamma$-GAGE analysis for seven gene sets representing four prehypothesized pathways

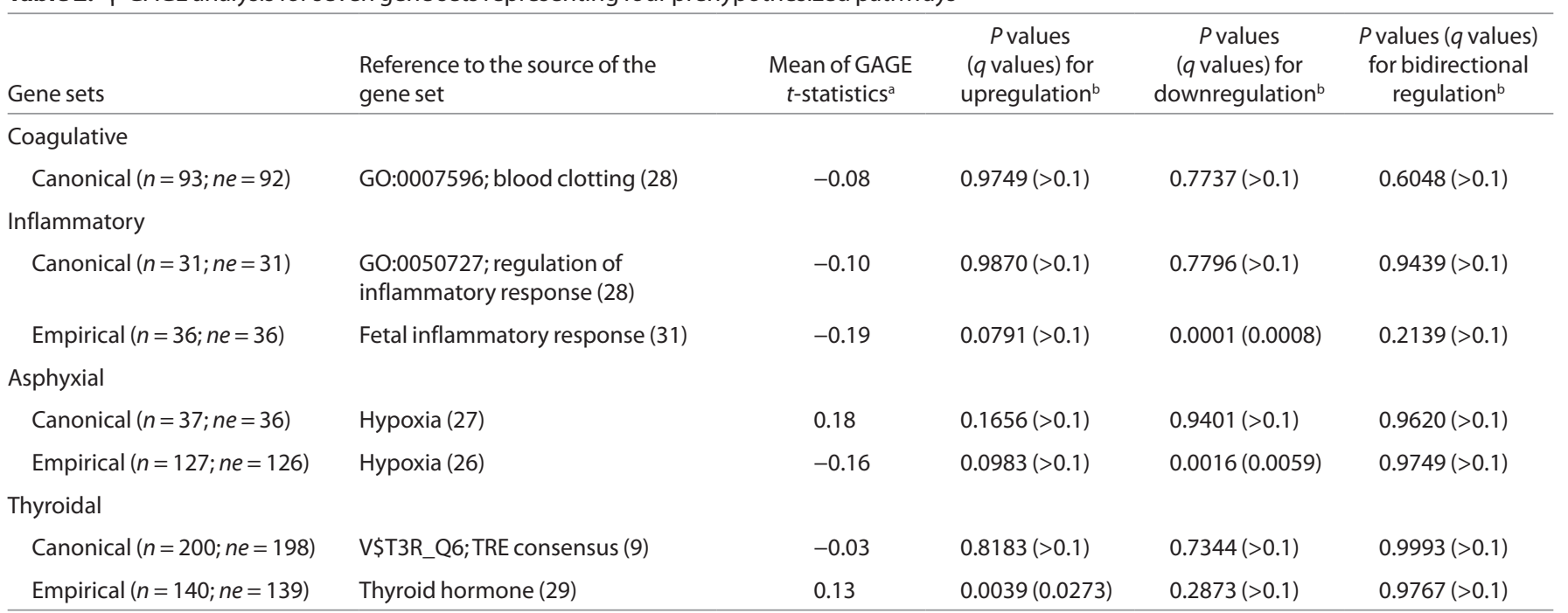

$n=$ Number of genes in the gene set; $n e=$ number of genes of the gene set that are found in the array used in this study.

FDR, false-discovery rate; GAGE, generally applicable gene set enrichment; TRE, thyroid-responsive element.

aBecause the number of genes in all of the seven selected gene sets is > 30, the GAGE $t$-statistic can be taken as a $z$-statistic. Therefore, the mean of GAGE $t$-statistics, which can be expressed in terms of SD units, is an approximation of effect size. ${ }^{b} \mathrm{Global} P$ values of all pairs, with $q$ values in parentheses for the three significant findings calculated to adjust for multiple testing using $q$ values $R$ package with FDR set at 0.05 .

differ between cases and controls. Cases and controls were broadly comparable in socioeconomic and demographic characteristics (Table 1).

\section{Analysis of Seven Preselected Gene Sets}

Each case-control pair received a score (expressed as a generally applicable gene set enrichment (GAGE) $t$-statistic) summarizing the case-control difference in the expression of all genes (from 31 to 200 genes, depending on the gene set). This score can be interpreted as the difference between cases and controls expressed as fractions of an SD. These score differences were then summarized across pairs and assessed for the statistical significance of the summarized difference, expressed as the global $P$ value for the contrast of cases and controls for each gene set.

Three gene sets, all empirical, showed a significantly different gene regulation across the population of case-control pairs 
a

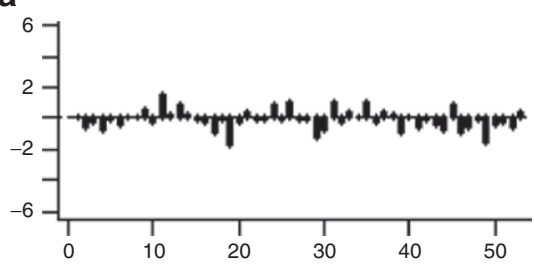

C

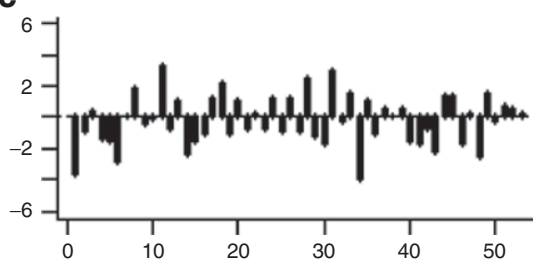

e

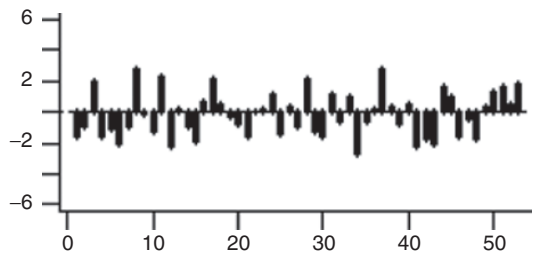

g

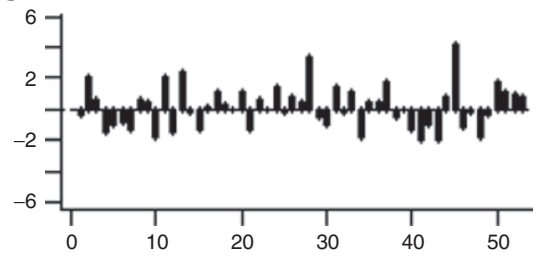

b

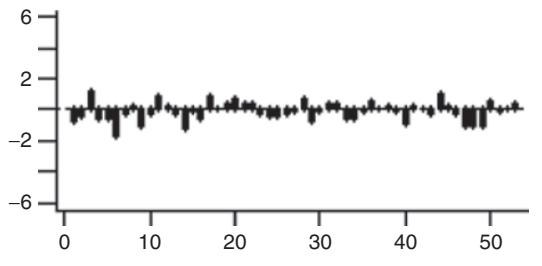

d

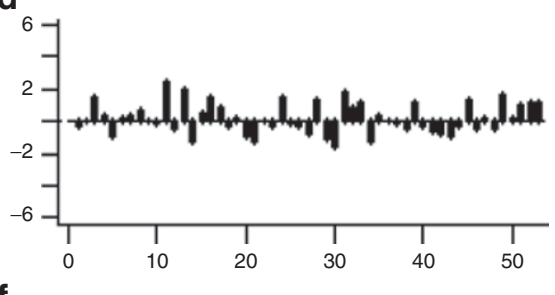

f

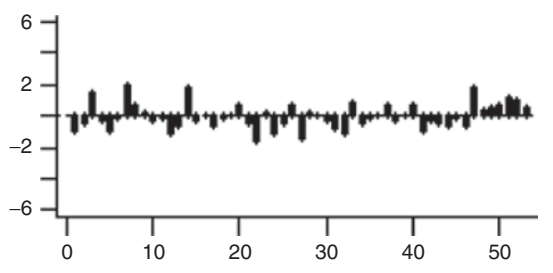

Figure 1. GAGE $t$-statistics for the seven prehypothesized gene sets. (a) Canonical coagulation, (b) canonical inflammation, (c) empirical inflammation, (d) canonical asphyxia, (e) empirical asphyxia, (f) canonical thyroid, and (g) empirical thyroid gene sets. For each graph: $x$-axis: matched pair (total 53 pairs); $y$-axis: scale of GAGE $t$-statistic; each bar within each graph: the GAGE $t$-statistic of the gene set for each pair. GAGE, generally applicable gene set enrichment.

(Table 2). The empirical inflammatory and asphyxial gene sets were both significantly downregulated in CP cases, as compared with controls, with effect sizes of -0.19 SD units and -0.16 SD units, respectively. The thyroidal gene set was significantly upregulated by $+0.13 \mathrm{SD}$ units. These three differences were highly significant either with ( $q$ value) or without ( $P$ value) correction for multiple testing.

The distribution of GAGE $t$-statistics for each of the gene sets, which illustrates the gene set differences across case-control pairs, is shown in Figure 1. The three significant gene sets showed many pairs with large interpair differences in gene expression, whereas the canonical hypoxic and thyroidal gene sets showed modest interpair differences. The coagulation gene set and the canonical inflammatory gene set showed virtually no differences. Heterogeneity across pairs in the direction of differential expression (either up or down) is notable for all three statistically significant gene sets (Table 2, Figure 1).

Individual Genes in the Fetal Inflammatory Response Syndrome Gene Set

The largest case-control differences in gene expression (0.19SD) were seen for the fetal inflammatory response syndrome (FIRS) gene set. Differences in gene expression are often described as "fold changes," i.e., the ratio of the degree of gene expression in cases as compared with controls, most conveniently expressed as the binary logarithm $\left(\log _{2} n=\log\right.$ arithm to the base 2$)$ of the fold change. Figure 2 shows the heat map of the $\log _{2}$ fold change of all genes in the FIRS gene set for all case-control pairs ordered by the magnitude of the GAGE $t$-statistics. The largest pair differences were seen in the following genes: S100A9 (S100 calciumbinding protein A9), S100A12 (S100 calcium-binding protein A12), ALOX5AP (arachidonate 5-lipoxygenase-activating protein), PGLYRP1 (peptidoglycan recognition protein 1), HP (haptoglobin), FLOT1 (flotillin 1), and FGR (Gardner-Rasheed feline sarcoma viral oncogene homolog) (Figure 2).

\section{Assessing Significant Gene Set Findings in CP Subsets}

The heterogeneity of GAGE $t$-statistics across pairs suggests the need to stratify on covariates such as gestational age and motor type. Table 3 shows the findings for each gene set for children born at $37 \mathrm{wk}$ or later ( $n$ pairs $=33$ ) and before $37 \mathrm{wk}$ ( $n$ pairs $=20)$ and for those with quadriplegia $(n$ pairs $=24)$, diplegia $(n$ pairs $=15)$, and hemiplegia $(n$ pairs $=13)$.

FIRS showed a clear interaction with gestational age: among 20 preterm pairs, FIRS was significantly upregulated in $\mathrm{CP}$ 
cases, whereas among 33 term-born pairs, FIRS was significantly downregulated among cases. The FIRS upregulation seen in preterm cases was paralleled by the upregulation in diplegic cases, who are dominantly preterm. In parallel, the strongest contribution to downregulation of inflammation

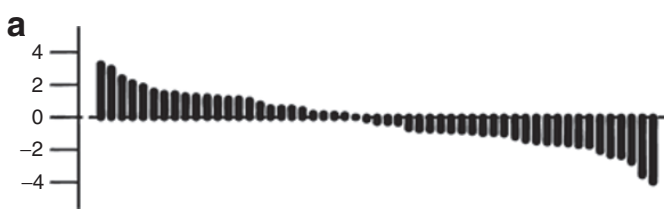

b

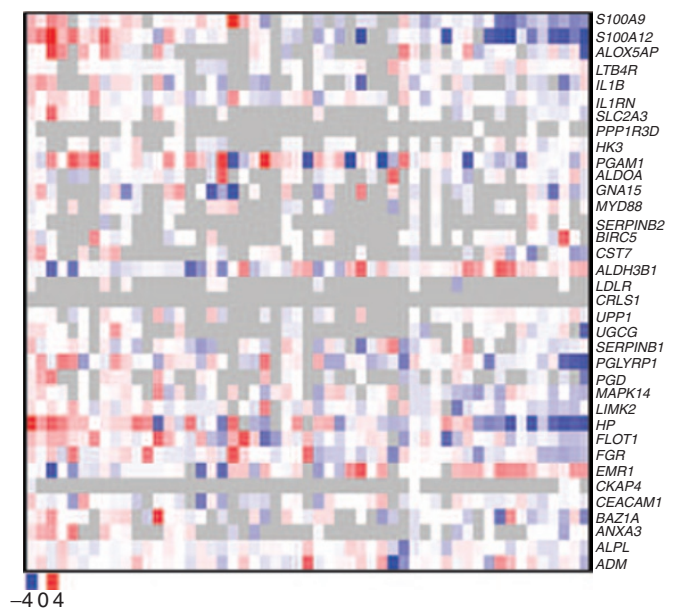

Figure 2. Heat map of FIRS gene set with pairs ordered by magnitude of GAGE $t$-statistics. (a) FIRS gene set in which the matched pairs are ordered by the values of the GAGE $t$-statistics of the pairs from most positive to most negative. (b) Heat map: $x$-axis: matched pairs in the same order as the upper graph; $y$-axis: gene names. Each small square represents $\log _{2}$ fold change of each of all genes of FIRS gene set of each of all pairs. Gradient scale for color from bluest (most negative $\log _{2}$ fold change or the gene expresses lowest in case vs. control) to white ( $\log _{2}$ fold change is zero or the gene expresses equally in case vs. control) to reddest (positive $\log _{2}$ fold change or the gene expresses highest in case vs. control): -4 to 0 to +4 . Gray color: absence of data (missing values) due to unmet filtering criteria. FIRS, fetal inflammatory response syndrome; GAGE, generally applicable gene set enrichment. came from hemiplegic cases $(n=13)$, who were nearly all born at term. Quadriplegia also showed downregulation of FIRS but not as strongly as hemiplegia.

The empirical hypoxic gene set also showed significant upregulation in preterm cases and the opposite with term cases. Downregulation of the hypoxic gene set was seen in hemiplegia and quadriplegia. The thyroidal upregulation signal was derived entirely from preterms and from children with quadriplegia. The sample size precluded consideration of interactions between gestational age and motor typology (Table 3).

\section{Kyoto Encyclopedia of Genes and Genomes Gene Sets}

We explored case-control differences for the 205 gene sets archived by the Kyoto Encyclopedia of Genes and Genomes (2009 version) (8). The five most upregulated gene sets in CP cases as compared with controls were ribosome, systemic lupus erythematosus, olfactory transduction, cell cycle, and oxidative phosphorylation. Downregulation was seen most strongly for three of the above-mentioned five gene sets, ribosome, cell cycle, and systemic lupus erythematosus, as well as for leukocyte transendothelial migration and regulation of actin cytoskeleton gene sets.

Using the approach of Storey $(10,11)$ to control for the falsediscovery rate, ribosome, systemic lupus erythematosus, and olfactory transduction were significantly upregulated; ribosome, leukocyte transendothelial migration, and regulation of actin cytoskeleton were significantly downregulated; and the ribosome gene set was significantly bidirectionally regulated.

The analysis for all individual genes available in the arrays reveals that no individual gene was significantly differentially expressed between cases and controls after adjusting for multiple testing. The lack of single gene expression differences confirms the value of gene set analysis for aggregating coordinated expression signals from related genes in gene sets in exploring pathophysiological pathways to disease (Table 4).

Table 3. Gene expression findings for three gene sets stratified by GA and CP type

\begin{tabular}{|c|c|c|c|c|c|c|c|c|c|}
\hline \multirow[b]{2}{*}{ Gene sets } & \multicolumn{3}{|c|}{ Empirical inflammatory gene set } & \multicolumn{3}{|c|}{ Empirical asphyxial gene set } & \multicolumn{3}{|c|}{ Empirical thyroidal gene set } \\
\hline & $\begin{array}{c}\text { Mean } \\
\text { GAGE } \\
\text { t-statistic }\end{array}$ & $\begin{array}{c}P \text { value } \\
\text { upregulation }\end{array}$ & $\begin{array}{c}P \text { value } \\
\text { downregulation }\end{array}$ & $\begin{array}{c}\text { Mean } \\
\text { GAGE } \\
t \text {-statistic }\end{array}$ & $\begin{array}{c}P \text { value } \\
\text { upregulation }\end{array}$ & $\begin{array}{c}P \text { value } \\
\text { downregulation }\end{array}$ & $\begin{array}{c}\text { Mean } \\
\text { GAGE } \\
t \text {-statistic }\end{array}$ & $\begin{array}{c}P \text { value } \\
\text { upregulation }\end{array}$ & $\begin{array}{c}P \text { value } \\
\text { downregulation }\end{array}$ \\
\hline $\begin{array}{l}\geq 37 w k \\
(n=33)\end{array}$ & -0.42 & 0.6567 & 0.00007 & -0.36 & 0.5014 & 0.00089 & -0.04 & 0.1345 & 0.1204 \\
\hline \multicolumn{10}{|l|}{ CP type ${ }^{a}$} \\
\hline $\begin{array}{l}\text { Quadriplegia } \\
(n=24)\end{array}$ & -0.26 & 0.60869 & 0.02830 & -0.18 & 0.11721 & 0.01024 & 0.08 & 0.01865 & 0.16745 \\
\hline $\begin{array}{l}\text { Diplegia } \\
(n=15)\end{array}$ & 0.16 & 0.03753 & 0.23559 & 0.03 & 0.27462 & 0.30122 & 0.32 & 0.10591 & 0.84832 \\
\hline
\end{tabular}

CP, cerebral palsy; GA, gestational age; GAGE, generally applicable gene set enrichment.

${ }^{a} \mathrm{CP}$ type missing for one case. 
Table 4. Most upregulated and most downregulated Kyoto Encyclopedia of Genes and Genomes gene sets in cases as compared with controls

\begin{tabular}{|c|c|c|c|c|c|c|c|c|}
\hline \multicolumn{3}{|c|}{ Most upregulated gene sets } & \multicolumn{3}{|c|}{ Most downregulated gene sets } & \multicolumn{3}{|c|}{ Most bidirectionally regulated gene sets } \\
\hline Gene set & $P$ value & $q$ value $^{\mathrm{a}}$ & Gene set & $P$ value & $q$ value $^{\mathrm{a}}$ & Gene set & $P$ value & $q$ value $^{\mathrm{a}}$ \\
\hline Ribosome & $4.4 \times 10^{-40}$ & $9.1 \times 10^{-38}$ & Ribosome & $4.1 \times 10^{-42}$ & $8.3 \times 10^{-40}$ & Ribosome & $1.9 \times 10^{-14}$ & $3.9 \times 10^{-12}$ \\
\hline От & $5.8 \times 10^{-05}$ & $3.9 \times 10^{-03}$ & RAC & $5.1 \times 10^{-04}$ & $3.5 \times 10^{-02}$ & APM & $4.9 \times 10^{-02}$ & $>0.1$ \\
\hline $\mathrm{CC}$ & $2.4 \times 10^{-03}$ & $>0.1$ & $\mathrm{CC}$ & $1.8 \times 10^{-03}$ & $9.5 \times 10^{-02}$ & PD & $5.0 \times 10^{-02}$ & $>0.1$ \\
\hline OP & $5.6 \times 10^{-03}$ & $>0.1$ & SLE & $9.2 \times 10^{-03}$ & $>0.1$ & MODY & $5.1 \times 10^{-02}$ & $>0.1$ \\
\hline
\end{tabular}

APM, aminophosphonate metabolism; BTF, basal transcription factors; CC, cell cycle; FDR, false-discovery rate; LTM, leukocyte transendothelial migration; MODY, maturity onset diabetes of the young; $\mathrm{OP}$, oxidative phosphorylation; $\mathrm{OT}$, olfactory transduction; PD, Parkinson's disease; RAC, regulation of actin cytoskeleton; SLE, systemic lupus erythematosus. ${ }^{a} q$ values were calculated using $q$ values with FDR level set at 0.05 .

\section{Quantitative PCR Validation of mRNA Data}

To validate our microarray findings, we used quantitative PCR (qPCR) techniques to examine the housekeeping genes ACTB ( $\beta$-actin) and PPIA (peptidylprolyl isomerase A), both commonly used in the literature to validate microarray findings. To validate genes differentially expressed, we selected FCGR2A (Fc fragment of IgG, low-affinity IIa receptor), a representative gene of the lupus pathway from the Kyoto Encyclopedia of Genes and Genomes database.

For $A C T B$ and PPIA genes, the correlation coefficient between the $\log _{2}$ intensity of microarray data and mean $C_{T}$ (cycle threshold) value of qPCR was $-0.52(P<0.0001)$. For FCGR2A, the correlation coefficient between the $\log _{2}$ intensity of microarray data and mean $\mathrm{C}_{\mathrm{T}}$ of $\mathrm{qPCR}$ was $-0.43(P$ $<0.0001$ ), and the correlation coefficient between $\log _{2}$ fold change of microarray data and $\log _{2}$ fold change of qPCR data was $0.38(P=0.0197)$.

\section{DISCUSSION}

\section{Principal Findings and Their Direction}

We found three gene sets, each reflecting a separate pathophysiological pathway, to be significantly dysregulated in newborns who later developed CP. The direction of the signals was not entirely as expected. Although the upregulation of inflammation noted among prematures later developing $\mathrm{CP}$ is consistent with several previous studies (12-14), the significant downregulation of inflammation in term CP and upregulation of thyroidal function in premature CP seem opposite to what might be expected on the basis of the literature $(15,16)$. However, dysregulation of a molecular pathway, as measured by changes in gene expression, can result from the influences of several types of cellular control processes, not all in the same direction. For example, mitochondrial defects leading to decreased energy output activate a transcriptional response to increase energy output, registered as increased expression of mitochondrial genes (17). It is thus more useful to consider dysregulation in either direction as indicating some perturbation of cellular control systems involved in the pathophysiological pathway of interest.

\section{Validity Studies of Archived Newborn Blood Spots}

In Michigan, spots were normally collected on day 1 or 2 of life, allowed to dry in ambient conditions for $4 \mathrm{~h}$, and sent to the Michigan Department of Community Health, where, after testing, they were stored in boxes at room temperature. Since 2009, with the establishment of the Michigan Biotrust for Health (18), all new specimens are stored at $-20^{\circ} \mathrm{C}$. None of the spots used in this study were frozen.

Four sources of evidence support the validity of the mRNA data obtained from our samples:

1 Published research by others: Several studies have shown that even after $20 \mathrm{y}$ of unfrozen storage, mRNA can be recovered from unfrozen blood spots (19-22).

2 Our own published work: We have published two papers demonstrating the reliability and validity of the archived unfrozen newborn blood spots we use in this study. Haak et al. (23) have demonstrated the replicability $(R>0.90)$ of microarray gene expression, and Resau et al. (24) have shown that sex-specific genes such as XIST and KDM5D are distinguishable in male and female archived unfrozen neonatal blood spots. A third paper by Khoo et al. (25) shows the high sensitivity of gene detection in blood spotted on filter paper with the Agilent $4 \times 44 \mathrm{~K}$ microarray system (Agilent Technologies, Santa Clara, CA) used in this study.

3 qPCR confirmation of microarray findings: In our earlier study (23), we showed that using qPCR, we could identify two housekeeping genes (HKI and MRPLII) and two inflammatory genes (ITGAX and NFKB-1) in archived unfrozen blood spots. As shown above, in the current study, we move beyond qPCR identification and show the strong correlation between the amount of mRNA detected by microarray and qPCR for another three genes.

4 Unpublished pilot data comparing frozen and unfrozen newborn blood spots: Microarray was performed in our laboratory on paired spots from the same newborns studied 10 y ago. Frozen $\left(-80^{\circ} \mathrm{C}\right)$ blood spots obtained from five Michigan newborns as part of a research study were compared with the blood spots archived by the state after genetic screening obtained at approximately the same age. The average Pearson correlation coefficient in total gene expression between frozen and unfrozen spots was 0.76 .

\section{Exploratory Gene Set Analyses}

Our exploratory analysis of the Kyoto Encyclopedia of Genes and Genomes gene set reinforced the importance of 
inflammatory processes in $\mathrm{CP}$, given that several gene sets related to such processes were significantly dysregulated. The lupus gene set, for example, contains genes such as those related to T-cell and B-cell receptor signaling pathways and cytokinecytokine receptor interaction. Several Kyoto Encyclopedia of Genes and Genomes inflammatory gene sets that differed in case-control expression showed the same interaction with gestational age as FIRS, with upregulation in preterm-born CP cases and downregulation in term-born CP cases.

\section{Sensitivity Analyses}

To determine whether values resulting from filtering procedures affect the results, we repeated the analyses with imputation for missing values and found nearly identical gene set findings. We also performed permutations on randomly selected gene sets of different sizes to evaluate the false-positive rate of the GAGE method on our actual microarray data. The probability of false-positive findings of the GAGE method is $<0.05$ when the significance level for the test is set at $<0.05$. (A detailed description is in the Supplementary Methods online.)

In conclusion, we found that archived unfrozen filter paper blood spots, even after many years of storage, retain enough mRNA to describe a distinct gene expression profile in neonates who later develop CP. The pathophysiological pathways we identify get us closer to understanding the changes that take place in the interval between prenatal or perinatal brain insult and the clinical manifestation of the CP syndrome. Our results also underline the scientific promise of using the large collections of state-archived newborn blood spots to develop compendia of expression profiles in disease states characterized by perturbations in gene expression that can be captured shortly after birth.

\section{METHODS}

\section{Study Subjects}

Children with clinically verified CP aged $2-15$ y $(n=53)$ were enrolled in specialty clinics in Lansing, Ann Arbor, and Grand Rapids, MI, if they had Gross Motor Function Classification System scores $>1$ and did not have a recognized malformation syndrome accounting for the $\mathrm{CP}$. Control subjects were enrolled in primary-care settings in Michigan and were individually matched on year of birth, sex, and gestational age in four categories $(<28,28-32,33-37$, and $>37 \mathrm{wk})$ and were free of major brain disorders. Birth certificates, maternal and infant hospital discharge abstracts, and residual newborn blood specimens were retrieved from the Michigan Department of Community Health, after obtaining written informed consent from the mother or guardian of the study subject. Case and control mothers were interviewed by telephone about reproductive exposures. Approval for this study was obtained from the institutional review boards of all participating institutions.

\section{RNA Isolation and CDNA Preparation}

RNA isolation, cDNA synthesis, and microarray analysis of blood spot samples was performed at the Laboratory of Microarray Technology at VARI (Van Andel Research Institute) per our published protocol (25). Three 3-mm blood spot punches were homogenized using a TissueLyser (Qiagen, Valencia, CA) before isolation using the illustra RNAspin Minikit (GEHealthcare, Buckinghamshire, United Kingdom). Deoxyribonuclease digestion was carried out during RNA isolation to remove any contaminating DNA in RNA. Total RNA was then concentrated with an RNA Clean \& Concentrator-5 Kit (Zymo Research, Orange, CA). RNA quality and quantity were evaluated using an RNA Pico Lab Chip on the Agilent BioAnalyzer. The average RNA integrity number was $2.3 \pm 0.71$. The WT-Ovation Pico RNA Amplification System (NuGEN Technologies,
San Carlos, CA), based on the Ribo-SPIA technology, was used to amplify total RNA and synthesize first- and second-stranded cDNA. Because this amplification system is initiated both at the $3^{\prime}$ end and randomly throughout the whole transcriptome, it has the advantage of amplifying non-poly A transcripts and compromised RNA.

\section{Gene Expression Microarray Assays}

For gene expression microarray, cDNA was labeled with Alexa Fluor 3 fluorescent dye from the BioPrime Total Genomic Labeling System (Invitrogen Life Technologies, Carlsbad, CA) before purification using the PureLink PCR Purification System (Invitrogen). Purified labeled product was then applied to a $8 \times 60,000$ whole human genome gene expression microarray (Agilent). Each array contains 60,000 oligonucleotide probes (60 bp probe) covering 27,958 Entrez gene RNAs and 7,419 long intergenic noncoding RNAs. The arrays were hybridized for $17 \mathrm{~h}$ at $65^{\circ} \mathrm{C}$ and $10 \mathrm{rpm}$ rotation speed, then washed for $2 \mathrm{~min}$ each with washing buffers 1 and 2 and scanned with an Agilent G3 highresolution scanner. Probe features were extracted from the microarray scan data using Feature Extraction software v. 10.7.3.1 (Agilent).

\section{Microarray Confirmation by qPCR}

Masked cDNA samples synthesized from neonatal blood spot RNA were used for qPCR analysis. Specific optimized Taqman probes and primers were obtained from Applied Biosystems by Life Technologies (Carlsbad, CA), and qPCR was performed using Applied Biosystems 7500 Fast RealTime PCR System. We examined the correlation between $\log _{2}$ intensity of microarray data and mean $\mathrm{C}_{\mathrm{T}}$ of $\mathrm{qPCR}$ data for all three genes, and for the FCGR2A gene, we also assessed the correlation between $\log _{2}$ fold change of microarray data and $-\log _{2}$ fold change of qPCR.

\section{Selection of Seven Gene Sets Representing Four Hypothesized Pathways to CP}

The empirical hypoxic gene set is based on the responses of cells in tissue culture exposed to hypoxemia as compared with normoxemia (26), whereas the canonical hypoxic gene set is based on the view that hypoxiainducible transcription factor binds a consensus DNA sequence termed the hypoxia-responsive element (27). A similar approach was used to construct the canonical thyroid-responsive element gene set (28). The experimentally derived thyroid gene set was isolated following human exposure to thyroid hormone (29). The canonical inflammation pathway is GO:005072 (inflammatory response) (30), whereas the empirical gene set is from an experiment in prematures with and without evidence of fetal inflammation and infection (31). The coagulation cascade was represented by GO:0007596 (blood clotting biological processes) (30).

\section{Statistical Methods}

Data processing and analysis were performed using open source statistical software R (http://www.r-project.org/, version 2.13.2). Unqualified spots were filtered using the method of Patterson et al. (32), where the expression data were removed whenever the gProcessed signal was less than twice the gProcessed signal error. Gene expression data were normalized using a between-array quantile normalization method (33) and further aggregated to the gene level using the mean of the expression signal of all probes of each gene. Differential expression of individual genes was examined with the moderated paired $t$ test (appropriate for matched pairs) of the linear model and an empirical Bayes method implemented in R package limma (34). The significance of gene expression was corrected for multiple testing, setting the false-discovery rate at 0.05 (10). We chose the GAGE method (35), the only published method applicable to a matched case-control study among several methods for gene set analysis $(36,37)$. A fuller description of the GAGE method is provided in the Supplementary Methods online. For case-control comparisons of clinical data, exact conditional logistic regression and paired $t$-tests were used for categorical variables and continuous variables, respectively.

\section{SUPPLEMENTARY MATERIAL}

Supplementary material is linked to the online version of the paper at http://www.nature.com/pr

\section{ACKNOWLEDGMENTS}

We appreciate the work of the members of the OWL (Outcome, Wellness and Life-History in Cerebral Palsy) study team, including Mary Jo 
Cooley Hidecker, Jaime Slaughter, Suzette Baez Vanderbeek, Joseph Bonner, Ariel Brovont, Mary Crawford, Matt Francis, Alice Stanley and Sainan Wei; referring physicians Zachary Dyme, Steven DeRoos, and Andrea Kuldanek; and collaborators Harry Hawkins and Glenn Copeland of the Michigan Department of Community Health. All authors participated in the manuscript preparation and editing. In addition, N.T.H. conceived of and performed the data analyses; K.F. chose the gene sets and advised on analysis; W.F. and Q.L. advised on statistical analysis; J.B. performed the qPCR analyses and advised on laboratory aspects of the study; S.K.K. performed the microarray analyses and advised on laboratory aspects of the study; M.L., E.H., J.W. and N.D. supervised and organized data collection in clinical settings, advised on clinical aspects of the study, and participated in discussions of findings and manuscript reviews; J.R. supervised the microarray work and N.P. conceived of the study, obtained its funding, and serves as principal investigator of the grant.

\section{STATEMENT OF FINANCIAL SUPPORT}

This work was funded by National Institutes of Health grant R01 NS055101 and the Van Andel Research Institute. N.T.H. is partially funded by the Vietnam Education Foundation.

Disclosure: The authors declared no conflict of interest.

\section{REFERENCES}

1. Bax M, Goldstein M, Rosenbaum P, et al.; Executive Committee for the Definition of Cerebral Palsy. Proposed definition and classification of cerebral palsy, April 2005. Dev Med Child Neurol 2005;47:571-6.

2. Paneth N, Hong T, Korzeniewski S. The descriptive epidemiology of cerebral palsy. Clin Perinatol 2006;33:251-67.

3. Blood collection on filter paper for neonatal screening programs. National Committee for Clinical Laboratory Standards. 2003;23:21. Approved standard 4th Ed. NCCL Document LA4-A4. (http://doh.sd.gov/NewbornScreening/Manual/bloodcollection.pdf).

4 GE Healthcare. Blood specimen collection and handling procedure. Neonatal screening. General Electric Company. (updated March 2010). Accessed 9 July 2012. (http://www.whatman.com/References/Neonatal\%20Screening\%20Cue\%20Card\%20LR\%20FINAL\%203.2.10.pdf).

5. Roberts GC, Dennis EA, Meadows DH, Cohen JS, Jardetzky O. The mechanism of action of ribonuclease. Proc Natl Acad Sci USA 1969;62:1151-8.

6. Liew CC, Ma J, Tang HC, Zheng R, Dempsey AA. The peripheral blood transcriptome dynamically reflects system wide biology: a potential diagnostic tool. J Lab Clin Med 2006;147:126-32.

7. Mohr S, Liew CC. The peripheral-blood transcriptome: new insights into disease and risk assessment. Trends Mol Med 2007;13:422-32.

8. KEGG: Kyoto Encyclopedia of Genes and Genomes. Accessed 30 November 2009. (http://www.genome.jp/kegg/).

9. Ashburner M, Ball CA, Blake JA, et al. Gene ontology: tool for the unification of biology. The Gene Ontology Consortium. Nat Genet 2000;25:25-9.

10. Storey JD. A direct approach to false discovery rates. J R Statist Soc B 2002;64:479-98.

11. Storey JD. The positive false discovery rate: a Bayesian interpretation and the q-value. Ann Statist 2003;6:2013-35.

12. Leviton A, Allred EN, Kuban KC, et al. Microbiologic and histologic characteristics of the extremely preterm infant's placenta predict white matter damage and later cerebral palsy. the ELGAN study. Pediatr Res 2010;67:95-101.

13. Yoon BH, Jun JK, Romero R, et al. Amniotic fluid inflammatory cytokines (interleukin-6, interleukin-1beta, and tumor necrosis factor-alpha), neonatal brain white matter lesions, and cerebral palsy. Am J Obstet Gynecol 1997;177:19-26.

14. Huang HC, Wang CL, Huang LT, et al. Association of cord blood cytokines with prematurity and cerebral palsy. Early Hum Dev 2004;77:29-36.

15. Wu YW, Escobar GJ, Grether JK, Croen LA, Greene JD, Newman TB. Chorioamnionitis and cerebral palsy in term and near-term infants. JAMA 2003;290:2677-84.
16. Reuss ML, Paneth N, Pinto-Martin JA, Lorenz JM, Susser M. The relation of transient hypothyroxinemia in preterm infants to neurologic development at two years of age. N Engl J Med 1996;334:821-7.

17. Klomp JA, Petillo D, Niemi NM, et al. Birt-Hogg-Dubé renal tumors are genetically distinct from other renal neoplasias and are associated with up-regulation of mitochondrial gene expression. BMC Med Genomics 2010;3:59.

18. Michigan Department of Community Health. Michigan BioTrust for Health. Accessed 30 March 2012. (http://www.michigan.gov/mdch/ 0,1607,7-132-2942_4911_4916_53246-209738--,00.html).

19. Karlsson H, Guthenberg C, von Döbeln U, Kristenssson K. Extraction of RNA from dried blood on filter papers after long-term storage. Clin Chem 2003;49(6 Pt 1):979-81.

20. Zhang YH, McCabe ER. RNA analysis from newborn screening dried blood specimens. Hum Genet 1992;89:311-4.

21. Matsubara Y, Ikeda H, Endo H, Narisawa K. Dried blood spot on filter paper as a source of mRNA. Nucleic Acids Res 1992;20:1998.

22. Gauffin F, Nordgren A, Barbany G, Gustafsson B, Karlsson H. Quantitation of RNA decay in dried blood spots during 20 years of storage. Clin Chem Lab Med 2009;47:1467-9.

23. Haak PT, Busik JV, Kort EJ, Tikhonenko M, Paneth N, Resau JH. Archived unfrozen neonatal blood spots are amenable to quantitative gene expression analysis. Neonatology 2009;95:210-6.

24. Resau JH, Ho NT, Dykema K, et al. Evaluation of sex-specific gene expression in archived dried blood spots (DBS). Int J Mol Sci 2012;13: 9599-608.

25. Khoo SK, Dykema K, Vadlapatla NM, et al. Acquiring genome-wide gene expression profiles in Guthrie card blood spots using microarrays. Pathol Int 2011;61:1-6.

26. Chi JT, Wang Z, Nuyten DS, et al. Gene expression programs in response to hypoxia: cell type specificity and prognostic significance in human cancers. PLoS Med 2006;3:e47.

27. Maynard MA, Ohh M. Von Hippel-Lindau tumor suppressor protein and hypoxia-inducible factor in kidney cancer. Am J Nephrol 2004;24:1-13.

28. Subramanian A, Tamayo P, Mootha VK, et al. Gene set enrichment analysis: a knowledge-based approach for interpreting genome-wide expression profiles. Proc Natl Acad Sci USA 2005;102:15545-50.

29. Clément K, Viguerie N, Diehn M, et al. In vivo regulation of human skeletal muscle gene expression by thyroid hormone. Genome Res 2002;12:281-91.

30 The Gene Ontology. Accessed 30 April 2010. (http://www.geneontology. org).

31. Madsen-Bouterse SA, Romero R, Tarca AL, et al. The transcriptome of the fetal inflammatory response syndrome. Am J Reprod Immunol 2010;63:73-92.

32. Patterson TA, Lobenhofer EK, Fulmer-Smentek SB, et al. Performance comparison of one-color and two-color platforms within the MicroArray Quality Control (MAQC) project. Nat Biotechnol 2006;24:1140-50.

33. Bolstad BM, Irizarry RA, Astrand M, Speed TP. A comparison of normalization methods for high density oligonucleotide array data based on variance and bias. Bioinformatics 2003;19:185-93.

34. Smyth GK. Limma: linear models for microarray data. In: Gentleman R, Carey V, Dudoit S, Irizarry R, Huber W, eds. Bioinformatics and Computational Biology Solutions Using $R$ and Bioconductor. New York: Springer, 2005:397-420.

35. Luo W, Friedman MS, Shedden K, Hankenson KD, Woolf PJ. GAGE: generally applicable gene set enrichment for pathway analysis. BMC Bioinformatics 2009;10:161.

36. Curtis RK, Oresic M, Vidal-Puig A. Pathways to the analysis of microarray data. Trends Biotechnol 2005;23:429-35.

37. Goeman JJ, Bühlmann P. Analyzing gene expression data in terms of gene sets: methodological issues. Bioinformatics 2007;23:980-7.

38. Alexander GR, Himes JH, Kaufman RB, Mor J, Kogan M. A United States national reference for fetal growth. Obstet Gynecol 1996;87:163-8. 